\title{
Gender differences in CPR-quality and use of automated external defibrillators
}

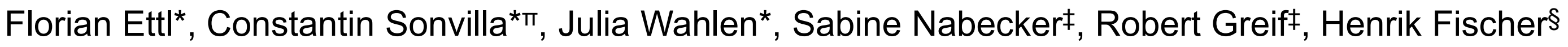 \\ ${ }^{*}$ Medical University of Vienna - Department of Emergency Medicine \\ 抽partment of Anaesthesiology and Pain Therapy, Bern University Hospital and University of Bern, Bern, Switzerland \\ $\S$ Federal Ministry of the Interior Herrengasse 7, 1014 Vienna, Austria
}

\section{Background and Goal of Study:}

Effective Basic Life Support (BLS) is crucial to improve survival after cardiac arrest. The early use of automated external defibrillators (AED) is a key link in the chain of survival. This study aimed to evaluate a possible gender difference in the quality of chest-compression-only (CCO) CPR and the handling of an AED.

\section{Materials and Methods:}

After ethics committee approval 445 first year medical students participated with signed informed consent in this open, prospective controlled trial.

Students performed single-rescuer CCO-CPR on a manikin (Ambu Man C, Ballerup, Denmark) for 4 minutes and followed the instructions of an AED (Defibtech Lifeline View).

Students' baseline demographics (e.g. gender, body mass index) were documented.

The combined parameter "effective compression ratio" $(E C R)^{1}$ assessed CPR-quality. Chest-compressions with correct depth, correct hand position and complete decompression multiplied by flow time (active CPR time) defines ECR. In the tested setting, correctly performed CCO-CPR has a maximum ECR of 0.92 .

In addition we recorded time-related parameters (e.g. flow time fraction) of the AED use. Students were asked to subjectively rate their own CPRquality on a 10-point Likert scale (NRS), where 1 means poor and 10 means excellent.

Data are presented as mean $\pm S D$. A $p$-value $<0.05$ was considered as significant.

\section{Results and Discussion:}

297 female (67\%) and 148 male (33\%) students participated. ECR differed significantly between gender (female: $0,13 \pm 0,20$, male: $0,08 \pm$ $0,14 ; p=0.001)$. The table shows the collected data.

The subjective CPR-quality rating was significantly higher in men $(7,0 \pm$ 1,6 versus $6,5 \pm 1,8$ for women, $p=0.001$ ).

\begin{tabular}{|l|r|r|r|}
\hline Parameter & \multicolumn{1}{l|}{$\begin{array}{l}\text { Male, } \\
\text { mean } \pm \text { SD }\end{array}$} & $\begin{array}{l}\text { Female, } \\
\text { mean } \pm \text { SD }\end{array}$ & p-value \\
\hline Compression rate, n/min & $99 \pm 14$ & $100 \pm 13$ & 0.52 \\
\hline Compression depth, mm & $52 \pm 10$ & $47 \pm 12 \mathrm{~mm}$ & $<0.001$ \\
\hline Wrong hand position, \% & $21,3 \pm 30,6$ & $21,9 \pm 30,9$ & 0.85 \\
\hline Incomplete decompression, \% & $50,2 \pm 38,2$ & $34,5 \pm 36,8$ & $<0.001$ \\
\hline Flow time fraction, \%, & $84,8 \pm 2,8$ & $83,2 \pm 8,5$ & $<0.004$ \\
\hline Body mass index (BMI) & $23,1 \pm 2,5$ & $20,8 \pm 2,6$ & $<0.001$ \\
\hline
\end{tabular}

\section{Conclusion:}

Women achieved significantly better CPR-quality (ECR), but they subjectively rated their own CPR-quality worse compared to men in that study. Further studies need to investigate the underlying reasons for these findings and this impact of gender on CPR-quality.

CPR trainers should be aware of that and address these gender differences during CPR training.
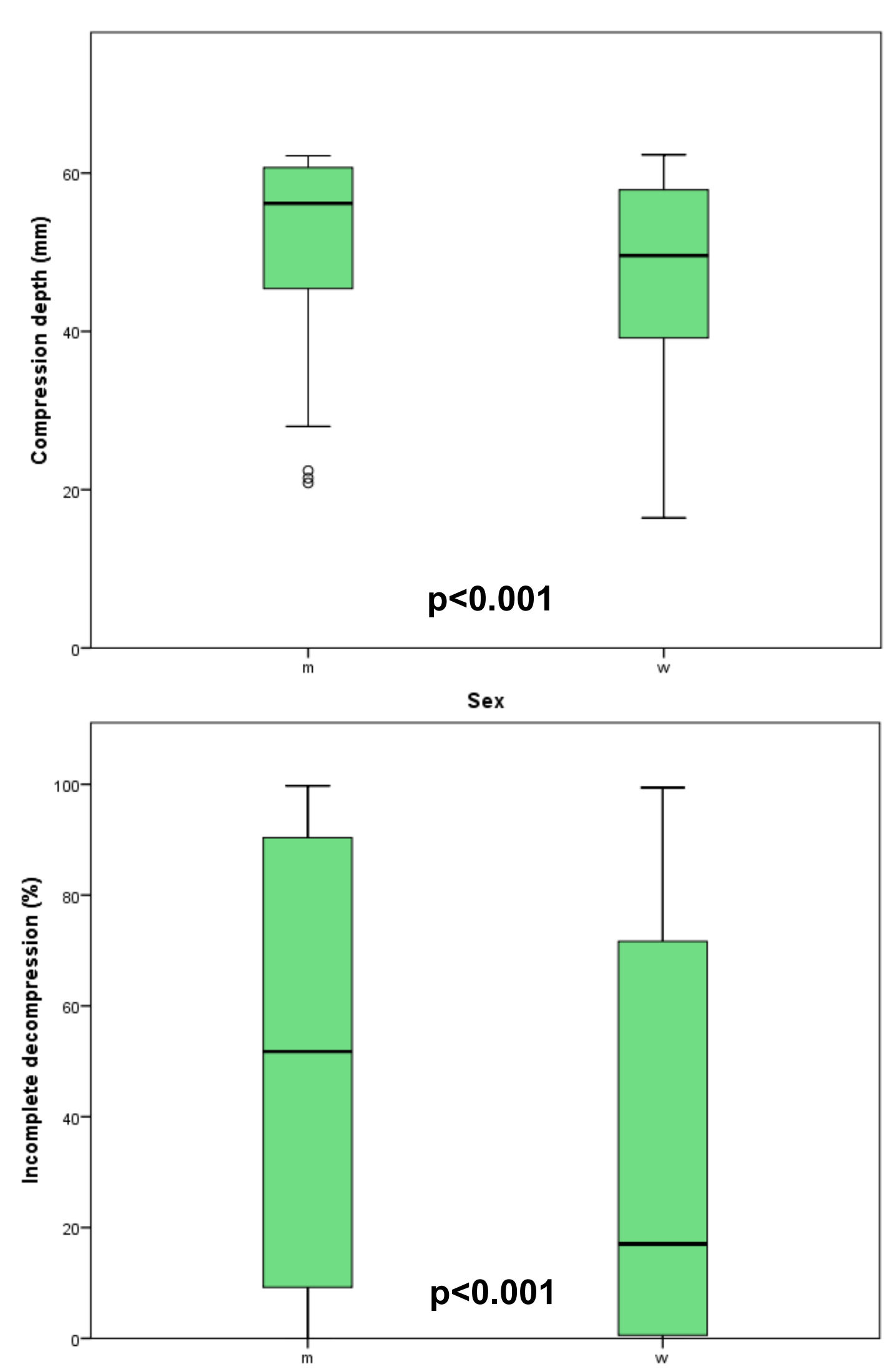

Sex

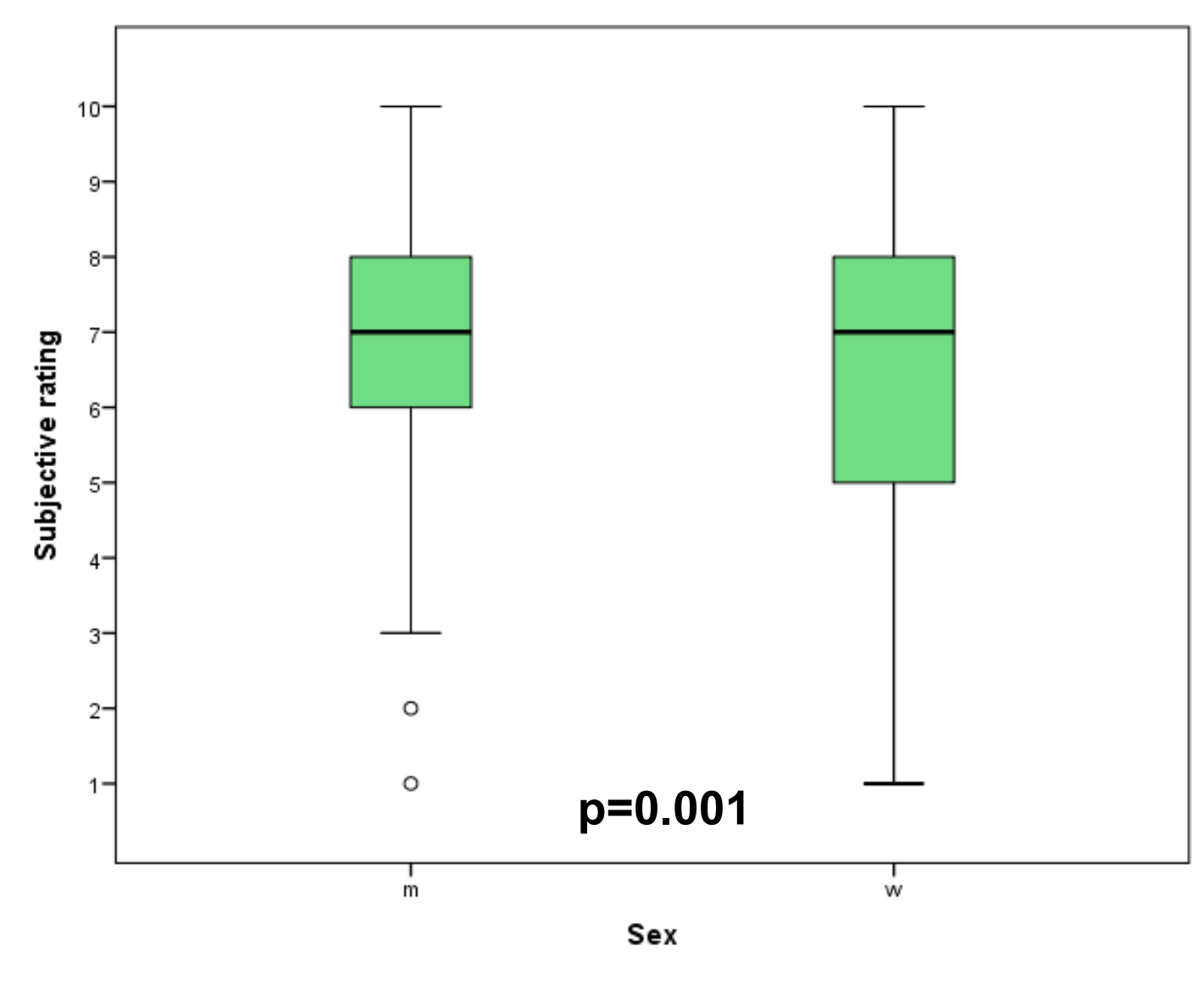

"Contact: Constantin Sonvilla n1242370@students.meduniwien.ac.at Department of Emergency Medicine Medical University of Vienna Währinger Gürtel 18-20/6D, 1090 Vienna, Austria 